\title{
Celiac disease in patients with systemic lupus erythematosus
}

\author{
Zahra Soltani $^{1}{ }^{\text {ID }}$, Azarakhsh Baghdadi ${ }^{1}$ ID , Mohammad Nejadhosseinian $^{1}$ ID , \\ Seyedeh Tahereh Faezi ${ }^{1}$ ID , Bijan Shahbazkhani $^{2}$ ID , Seyed Ali Mousavi ${ }^{1}$ ID , Kiarash Kazemi $^{1}$ ID \\ ${ }^{1}$ Rheumatology Research Center, Tehran University of Medical Sciences, Iran \\ ${ }^{2}$ Department of Gastroenterology and Liver Diseases, Imam Khomeini Hospital Complex, Tehran University of Medical Sciences, Iran
}

\begin{abstract}
Objectives: Celiac disease (CD) is one of the most common chronic diseases. Celiac disease has been associated with several autoimmune disorders, but the association with systemic lupus erythematosus (SLE) as a systemic autoimmune disease is still controversial. In this study, we aimed to determine the prevalence of biopsy-proven CD in patients with SLE, and to determine the clinical symptoms and laboratory data in these patients.

Material and methods: In a cross-sectional study, SLE patients at a referral clinic were evaluated for gastrointestinal symptoms between March and December 2016. Patients were evaluated by a gastroenterologist, and upper gastrointestinal endoscopy with intestinal biopsy was performed if deemed necessary. The clinical symptoms, laboratory data, and endoscopy results were recorded and compared between groups.

Results: In total, 130 patients were evaluated in this study. Gastrointestinal symptoms were present in $40 \%$ of the patients. Endoscopy was performed in all SLE patients with gastrointestinal symptoms. Four patients (3\%) were diagnosed as having CD based on biopsy results and response to a gluten-free diet. Anti-endomysium antibody (AEA) was found to be $100 \%$ sensitive and $99.2 \%$ specific for the diagnosis of CD in SLE patients, and anti-gliadin antibody (AGA) had a 50\% sensitivity and $98 \%$ specificity. Patients with comorbid CD and SLE were significantly more likely to have diarrhea, abdominal pain, nausea/vomiting, recurrent oral aphthosis, and anemia.

Conclusions: The results of this study suggest that a significant association is present between CD and SLE. We found a prevalence of 3\% for biopsy-proven CD in patients with SLE, which is five times the prevalence of $C D$ in the general population.
\end{abstract}

Key words: autoimmune disease, systemic lupus erythematosus, celiac disease, gluten-sensitive enteropathy.

\section{Introduction}

As a multisystemic disease, systemic lupus erythematosus (SLE) affects every organ in the body. While cutaneous, musculoskeletal, renal, and central nervous system manifestations are more common, gastrointestinal (GI) symptoms are also present in at least half of the patients with SLE [1-3].

Non-specific Gl symptoms, such as nausea and vomiting, anorexia, and abdominal pain, are the most prevalent, but severe, life-threatening complications can also occur $[4,5]$. A thorough evaluation is imperative to dis- tinguish among common etiologies of Gl symptoms in SLE, which can include adverse effects of medications, infections, SLE-induced manifestations, and comorbid GI disorders [6-8].

Celiac disease (CD) is one of the most common chronic diseases worldwide, with a prevalence of $1 \%$ globally. There is some evidence that the prevalence of CD might actually be rising [9]. Clinical manifestations of CD are variable, including gastrointestinal and non-gastrointestinal symptoms, which are elicited by gluten in genetically susceptible individuals [9]. 
Occurrence of multiple autoimmune disorders in a patient is a well-described phenomenon, although the exact mechanism is still not understood. There is clear evidence that CD and type 1 diabetes mellitus are strongly associated, with CD occurring in $4.4-11 \%$ of diabetic patients $[9,10]$.

Addison's disease is also closely related to CD, and patients with CD have been found to be 11.4 times more likely to develop Addison's disease [11]. Celiac disease has also been linked to thyroid disorders and autoimmune hepatitis [9].

Among the rheumatologic disorders, CD has been linked to Sjögren's syndrome (SS). The prevalence of CD in adult SS patients has been reported as high as 14\% [12]. The high prevalence of HLA-DQ2 haplotype in SS patients is presumably the root cause of this association $[9,12]$. An association between SLE and CD has also been suggested in many studies [13-16], although the evidence is limited and inconclusive $[9,17]$.

This study was, therefore, designed to evaluate the association between CD and SLE. Our primary goa was to assess whether patients with SLE have a higher prevalence of $C D$. Also, we sought to determine the symptoms of patients with concurrent CD and SLE, and to compare these symptoms with isolated SLE. Finally, we aimed to evaluate the effect of a gluten-free diet on disease activity in patients with comorbid CD and SLE.

\section{Material and methods}

In a cross-sectional study, patients who visited a referral SLE clinic at Shariati Hospital, Tehran University of Medical Sciences, Tehran, from March to December 2016 were evaluated.

Patients were diagnosed with SLE based on the American College of Rheumatology (ACR) criteria [18]. Patients were invited to participate in the study after a thorough discussion about the goals and methods of this study. If patients were interested, a written informed consent form was signed. A form was then filled out by a trained physician, including demographic data and gastrointestinal symptoms, and personal or family history of CD.

Patients were then visited by a fellowship-trained gastroenterologist, who decided whether the patient needed an upper Gl endoscopy and further evaluation for CD. Upper Gl endoscopy included a biopsy of the duodenum. The results of the endoscopic examination and intestinal biopsy were recorded. A diagnosis of CD was only made after a pathologist reported the results of the biopsy, which was also graded according to the Marsh classification [19].

Laboratory tests were ordered for all patients if deemed necessary. Otherwise, the results of laboratory tests in the last 6 months were recorded if available.
Laboratory tests of interest were a complete blood count, erythrocyte sedimentation rate (ESR), C-reactive protein (CRP), plasma creatinine, total IgA, anti-endomysium (AEA) and anti-gliadin (AGA) antibodies, complement studies, disease-specific antibodies, and relevant HLA studies. Systemic lupus erythematosus activity was scored and recorded based on the Systemic Lupus Erythematous Disease Activity Index (SLEDAI) [20].

The ethics committee of our institution (Tehran University of Medical Sciences) reviewed and approved the study protocol prior to initiating the study (No. 02/1398, date of receipt: 5/5/2019).

An a priori power analysis (G*Power 3.1) was performed for sample size estimation. With a 0.01 prevalence of $C D, \alpha=0.05$, and power $=0.8$, the projected total sample size was calculated to be 130 patients. Statistical analysis was done with IBM SPSS Statistics for Windows, Version 25.0 (Armonk, NY). As this was not a comparative study, descriptive statistics were used to report means, ranges, and standard deviations.

Descriptive statistics were used for frequencies and means. Student's t-test was used to measure differences in means when the data were normally distributed, and the Mann-Whitney $U$ test was used to measure differences in means when the data were non-normally distributed. The $\chi^{2}$ test was used to identify differences in categorical variables. A $p$-value of $<0.05$ was considered significant.

\section{Results}

A total of 130 patients were included in the final analysis, with a mean age of 31.5 years (SD, 8.3 years, range, 16-60). There were 106 (81.5\%) female and $24(18.5 \%)$ male patients in this study. Patients were diagnosed and treated for SLE for a mean of 4.6 years (SD, 5.6 years, range, 0.17-30 years) prior to this study. The mean SLEDAI was 2.52 (range, 0-35, SD, 0.44).

Gastrointestinal symptoms were present in 52 (40\%) patients. Dyspepsia, anorexia, and abdominal pain were the most common symptoms. None of the patients had bloody diarrhea or steatorrhea.

An upper $\mathrm{Gl}$ endoscopy and intestinal biopsy were done in 49 (38\%) patients, based on the evaluation performed by a fellowship-trained gastroenterologist. Endoscopic findings were normal in 29 patients (59.2\%). Erosive esophagitis was found in 14 patients (28.5\%), a gastric or duodenal ulcer was each found in 2 patients (4.1\%), and erosive gastritis was found in 1 patient (2\%).

Four patients were diagnosed with CD after pathology specimens were reviewed, which equals a $3 \%$ prevalence of CD in SLE patients in this study, of which three were female. The diagnosis was also confirmed by clinical improvement following a gluten-free diet. All 
four patients had recurrent oral aphthous ulcers. Also, all four had positive AEA, and two had positive AGA antibodies. All patients with CD had a positive HLA-DQ8, while only one was positive for HLA-DQ2. We, therefore, found a sensitivity and specificity of $100 \%$ and $99.2 \%$ for a positive AEA in the diagnosis of CD in patients with SLE, and a sensitivity and specificity of $50 \%$ and $98.4 \%$ for AGA.

Two of the biopsy specimens had Marsh II changes, one had Marsh I changes, and one showed Marsh III pathologies. Two of the four patients with CD had villous atrophy on gross examination. Another patient had jejunal involvement. Patients with CD were significantly more likely to have abdominal pain $(p=0.02)$, diarrhea ( $p<0.001)$, dyspepsia $(p=0.002)$, and nausea/vomiting ( $p=0.025)$, which was not the case with other GI symptoms (all $p$-values $>0.05$ ).

There was no significant association between gender and CD $(p=0.73)$. The mean age of patients with and without a final diagnosis of CD was also statistically similar ( 29 vs. 29.8 years old, $p=0.53$ ). The mean time from the diagnosis of SLE was not significantly different between patients with and without CD (2.6 vs. 2.3 years, $p=0.7$ ).

Additionally, we did not find a significant difference in SLEDAI scores between patients with and without $C D(p=0.18)$. Patients with and without CD were not significantly different in having pathologic endoscopic findings $(p=0.656)$. Comparisons between patients with and without CD are summarized in Table I.

Our a priori goals in this study included evaluating the results of a gluten-free diet in the SLE disease activity. However, as all four patients with CD had controlled, inactive SLE, this was not possible.

\section{Discussion}

This study was performed to add to the body of evidence regarding the association between CD and SLE. We found a 3\% prevalence of biopsy-proven CD in 130 patients with SLE, which, at five times the national prevalence of CD in the general population [21], suggests a significant association between CD and SLE.

There is controversy in the literature regarding the association between SLE and CD. This association was first suggested by multiple authors in case reports [13-16]. The overlap in clinical and laboratory findings between standalone SLE and CD makes the clinical judgment of the physician extremely important in suspecting comorbid SLE and CD. Furthermore, GI manifestations of SLE cover a range of symptoms, which can also be difficult to distinguish from CD.

Rensch et al. [17], in a similar study, evaluated 103 patients to determine the prevalence of CD in SLE patients.
Table I. Comparisons between patients with and without a final diagnosis of celiac disease

\begin{tabular}{|c|c|c|c|}
\hline Variable & $\begin{array}{l}\text { CD group } \\
(n=4)\end{array}$ & $\begin{array}{l}\text { Non-CD group } \\
\quad(n=126)\end{array}$ & $p$-value \\
\hline \multicolumn{4}{|l|}{ Symptoms, $n$ (\%) } \\
\hline Diarrhea & $3(75)$ & $9(7.1)$ & $<0.001$ \\
\hline Constipation & $1(25)$ & $6(4.8)$ & 0.077 \\
\hline Fatigue & $2(50)$ & $19(15)$ & 0.062 \\
\hline Weight loss & $1(25)$ & $11(8.7)$ & 0.268 \\
\hline Dyspepsia & $4(100)$ & $36(28.6)$ & 0.002 \\
\hline $\begin{array}{l}\text { Nausea/ } \\
\text { vomiting }\end{array}$ & $1(25)$ & $4(3.2)$ & 0.025 \\
\hline $\begin{array}{l}\text { Abdominal } \\
\text { pain }\end{array}$ & $3(75)$ & $30(23.8)$ & 0.021 \\
\hline Bloating & $2(50)$ & $20(15.9)$ & 0.073 \\
\hline $\begin{array}{l}\text { Recurrent oral } \\
\text { aphthosis }\end{array}$ & $4(100)$ & $11(8.7)$ & $<0.001$ \\
\hline Atopy & $1(25)$ & $0(0)$ & $<0.001$ \\
\hline \multicolumn{4}{|l|}{ Laboratory tests } \\
\hline Positive AEA, $n(\%)$ & $4(100)$ & $1(0.8)$ & $<0.001$ \\
\hline Positive AGA, $n(\%)$ & $2(50)$ & $2(1.6)$ & $<0.001$ \\
\hline HLA-DQ2, $n(\%)$ & $1(25)$ & $4(3.2)$ & 0.025 \\
\hline HLA-DQ8, n (\%) & $4(100)$ & $9(7.1)$ & $<0.001$ \\
\hline Total lgA [mg/dl] & 230.5 & 130.2 & 0.049 \\
\hline $\mathrm{ESR}[\mathrm{mm} / \mathrm{h}]$ & 20.67 & 10.99 & 0.023 \\
\hline $\mathrm{CRP}[\mathrm{mg} / \mathrm{l}]$ & 11.33 & 4.69 & 0.028 \\
\hline Creatinine $[\mathrm{mg} / \mathrm{dl}]$ & 1.03 & 1 & 0.6 \\
\hline $\mathrm{Hb}[\mathrm{g} / \mathrm{dl}]$ & 9.87 & 11.88 & 0.004 \\
\hline $\begin{array}{l}\text { Platelets } \\
{\left[\text { count } / \mathrm{mm}^{3}\right]}\end{array}$ & $206 \times 10^{3}$ & $193 \times 10^{3}$ & 0.418 \\
\hline
\end{tabular}

Statistically significant comparisons are indicated in bold. AEA - anti-endomysium antibodies, AGA - anti-gliadin antibodies, $C D$ - celiac disease, $C R P-C$-reactive protein, $E S R$ - erythrocyte sedimentation rate, $\mathrm{Hb}$ - hemoglobin, HLA - human leukocyte antigen.

They found a $23 \%$ prevalence of a positive AGA, but none of their patients was positive for AEA. In the endoscopic examination of the AGA positive patients, none had changes compatible with CD. They concluded that AGA is $77 \%$ specific in the diagnosis of CD in patients with SLE.

In this study, we found AEA to have a $100 \%$ sensitivity and $99.2 \%$ specificity for the diagnosis of CD in SLE patients. Although AGA had a lower performance similar to previous studies, we did find a high specificity for AGA, owing to the very low prevalence of positive AGA in non-celiac SLE patients (1.58\%).

Our results were in line with a previous report by Marai et al. [22], which found a 3\% prevalence of AGA antibodies in 100 SLE patients. In another large matched 
case-control study, Dahan et al. [23] found a 0.8\% prevalence of CD in patients with SLE, which was higher than in non-CD patients (0.2\%).

The association between CD and SLE could be evaluated from another perspective. Freeman et al. [24] assessed 246 patients with CD and found a $2.4 \%$ prevalence of comorbid SLE and CD. Their findings suggested that SLE occurs far more frequently in patients with CD. While we used the opposite approach in this study, we also believe that comorbidity of CD and SLE is more common than is currently appreciated, and clinicians should be aware of the association between these two diseases.

In a large population-based study in Sweden, Ludvigsson et al. [25] evaluated the risk of SLE in patients with biopsy-proven CD. Their findings indicate a threefold increase in the risk of SLE in patients with CD compared to the general population. The association between SLE and CD is best understood as part of the "shared autoimmunity" concept [26].

Altered gene expression patterns are commonly shared between patients with a spectrum of autoimmune disorders, including several HLA and non-HLA genes [27] Furthermore, innate immune system activation through Toll-like receptors (TLRs), leading to overproduction of interferon type I, is another possible mechanism for the association between SLE and CD. The local inflammatory response caused by skin lesions (dermatitis herpetiform) in CD might also lead to overactivation of the immune system and subsequent SLE $[1,25]$.

Based on our findings, we agree with previous authors that routine serological testing for $C D$ is not recommended in SLE patients unless clinical suspicion is present.

In this study, we also aimed to determine the clinical and laboratory characteristics of comorbid CD and SLE. As expected, diarrhea was significantly more common in patients with SLE. Although the statistical significance was also observed for dyspepsia, nausea/vomiting, and abdominal pain, none of the clinical findings were sensitive and/or specific enough to be an excellent predictor of CD.

With the rising popularity of gluten-free diets (GFD) without a medical indication, an informative discussion regarding gluten and a GFD is recommended. Unless the patient is medically diagnosed with CD, eliminating gluten from the diet is an ill-advised decision, which not only imposes an undue economic burden on the patient, but also deprives the patient of a good protein source, especially in SLE patients who are already at a high risk of malnutrition [28, 29].

Further, the overall risk of CD is low in patients with SLE, and therefore a gluten-free diet is only indicated in patients with proven $C D$ with the recommendation of the treating physician.
Extraintestinal manifestations of CD are common and highly variable. However, in this study, we found that recurrent oral aphthosis has a 100\% sensitivity and 91\% specificity for the diagnosis of CD in SLE patients. Discriminating laboratory tests included total IgA, elevated ESR and CRP, and anemia.

It should be noted that CD should always be suspected in patients with chronic anemia non-responsive to treatment and should not be considered normal in well-controlled SLE.

We also aimed to evaluate the effect of a gluten-free diet on SLE disease activity. However, due to an inactive disease in all four CD patients, this was not possible. Further studies are needed to evaluate this effect.

We acknowledge limitations to this study. Our study population consists mainly of patients who have had well-controlled SLE, and have been under our care for some time. Therefore, we cannot infer the prevalence of Gl symptoms and comorbid CD in newly diagnosed patients or uncontrolled SLE.

Also, Gl symptoms are common in the general population, and significant recall bias might be in play here. Furthermore, we could not evaluate the effects of a gluten-free diet on SLE disease activity, as mentioned above.

The strengths of this study include the large study population, diagnosis of $C D$ based on biopsy results, evaluating symptoms and laboratory data and comparing them between groups, and performing an a priori power analysis.

\section{Conclusions}

We found a prevalence of 3\% for biopsy- proven celiac disease in patients with SLE, which strongly suggests a significant association between these two diseases. As the clinical manifestations of CD are highly variable, a high level of suspicion is needed when assessing patients with concurrent autoimmune disorders.

While routine screening for CD is not recommended in SLE patients, comorbid CD should be suspected in SLE patients with long-standing diarrhea, extraintestinal manifestations of CD (such as recurrent oral aphthosis), positive AGA and AEA antibodies, and anemia unresponsive to medical treatment in the presence of a wellcontrolled disease.

The authors declare no conflict of interest.

\section{References}

1. Alves SC, Fasano S, Isenberg DA. Autoimmune gastrointestinal complications in patients with systemic lupus erythematosus: case series and literature review. Lupus 2016; 25: 1509-1519, DOI: $10.1177 / 0961203316655210$. 
2. Fava A, Petri M. Systemic lupus erythematosus: diagnosis and clinical management. J Autoimmun 2019; 96: 1-13, DOI: 10.1016/j.jaut.2018.11.001.

3. Fawzy M, Edrees A, Okasha $H$, et al. Gastrointestinal manifestations in systemic lupus erythematosus. Lupus 2016; 25 : 1456-1462, DOI: 10.1177/0961203316642308.

4. Brewer BN, Kamen DL. Gastrointestinal and hepatic disease in systemic lupus erythematosus. Rheum Dis Clin North Am 2018; 44: 165-175, DOI: 10.1016/j.rdc.2017.09.011.

5. Ebert EC, Hagspiel KD. Gastrointestinal and hepatic manifestations of systemic lupus erythematosus. J Clin Gastroenterol 2011; 45: 436-441, DOI: 10.1097/MCG.0b013e31820f81b8.

6. Kröner PT, Tolaymat OA, Bowman AW, et al. Gastrointestinal manifestations of rheumatological diseases. Am J Gastroenterol 2019; 114: 1441-1454, DOI: 10.14309/ajg.0000000000000260.

7. Li Z, Xu D, Wang Z, et al. Gastrointestinal system involvement in systemic lupus erythematosus. Lupus 2017; 26: 1127-1138, DOI: 10.1177/0961203317707825.

8. Metry AM, Al Salmi I, Al Balushi F, et al. Systemic lupus erythematosus: symptoms and signs at initial presentations. Antiinflamm Antiallergy Agents Med Chem 2019; 18: 142-150, DOI: $10.2174 / 1871523018666181128161828$.

9. Denham JM, Hill ID. Celiac disease and autoimmunity: review and controversies. Curr Allergy Asthma Rep 2013; 13: 347-353, DOI: 10.1007/s11882-013-0352-1.

10. Shahbazkhani B, Faezi T, Akbari M, et al. Coeliac disease in Iranian type I diabetic patients. Dig Liver Dis 2004; 36: 191-194, DOI: 10.1016/j.dld.2003.10.015.

11. Elfström P, Montgomery SM, Kämpe O, et al. Risk of primary adrenal insufficiency in patients with celiac disease. J Clin Endocrinol Metab 2007; 92: 3595-3598, DOI: 10.1210/jc.2007-0960.

12. Luft LM, Barr SG, Martin LO, et al. Autoantibodies to tissue transglutaminase in Sjögren's syndrome and related rheumatic diseases. J Rheumatol 2003; 30: 2613-2619.

13. Komatireddy GR, Marshall JB, Aqel R, et al. Association of systemic lupus erythematosus and gluten enteropathy. South Med J 1995; 88: 673-676, DOI: 10.1097/00007611-19950600000018.

14. Mukamel M, Rosenbach Y, Zahavi I, et al. Celiac disease associated with systemic lupus erythematosus. Isr J Med Sci 1994; 30: 656-658.

15. Peña AS. Systemic lupus erythematosus, Sjögren's syndrome, and purpura in a patient with coeliac disease. Neth J Med 1987; 31: 305.

16. Rustgi AK, Peppercorn MA. Gluten-sensitive enteropathy and systemic lupus erythematosus. Arch Intern Med 1988; 148 1583-1584.
17. Rensch MJ, Szyjkowski R, Shaffer RT, et al. The prevalence of celiac disease autoantibodies in patients with systemic lupus erythematosus. Am J Gastroenterol 2001; 96: 1113-1115, DOI: 10.1111/j.1572-0241.2001.03753.x.

18. Yu C, Gershwin ME, Chang C. Diagnostic criteria for systemic lupus erythematosus: a critical review. J Autoimmun 2014; 48: 10-13, DOI: 10.1016/j.jaut.2014.01.004.

19. Marsh MN. Gluten, major histocompatibility complex, and the small intestine: a molecular and immunobiologic approach to the spectrum of gluten sensitivity ("celiac sprue"). Gastroenterology 1992; 102: 330-354.

20. Gladman DD, Ibañez D, Urowitz MB. Systemic Lupus Erythematosus Disease Activity Index 2000. J Rheumatol 2002; 29: 288-291.

21. Shahbazkhani B, Malekzadeh R, Sotoudeh $M$, et al. High prevalence of coeliac disease in apparently healthy Iranian blood donors. Eur J Gastroenterol Hepatol 2003; 15: 475-478, DOI: 10.1097/01.meg.0000059118.41030.96.

22. Marai I, Shoenfeld Y, Bizzaro N, et al. IgA and IgG tissue transglutaminase antibodies in systemic lupus erythematosus. Lupus 2004; 13: 241-244, DOI: 10.1191/0961203304lu1004oa.

23. Dahan S, Shor DB, Comaneshter D, et al. All disease begins in the gut: celiac disease co-existence with SLE. Autoimmun Rev 2016; 15: 848-853, DOI: 10.1016/j.autrev.2016.06.003.

24. Freeman HJ. Adult celiac disease followed by onset of systemic lupus erythematosus. J Clin Gastroenterol 2008; 42: 252-255, DOI: 10.1097/MCG.0b013e31802e70a1.

25. Ludvigsson JF, Rubio-Tapia A, Chowdhary V, et al. Increased risk of systemic lupus erythematosus in 29,000 patients with biopsy-verified celiac disease. J Rheumatol 2012; 39: 1964-1970, DOI: 10.3899/jrheum.120493.

26. Alarcón-Segovia D. Shared autoimmunity: the time has come. Curr Rheumatol Rep 2004; 6: 171-174, DOI: 10.1007/s11926004-0063-7.

27. Aune TM, Maas K, Parker J, et al. Profiles of gene expression in human autoimmune disease. Cell Biochem Biophys 2004; 40: 81-96, DOI: 10.1385/CBB:40:2:081.

28. Diez-Sampedro A, Olenick M, Maltseva T, Flowers M. A glutenfree diet, not an appropriate choice without a medical diagnosis. J Nutr Metab 2019; 2019: 2438934, DOI: 10.1155/2019/ 2438934.

29. Saturni L, Ferretti G, Bacchetti T. The gluten-free diet: safety and nutritional quality. Nutrients 2010; 2: 16-34, DOI: 10.3390/ nu20100016. 